\title{
Using the OLS Method to Analyze Indian Currency Fluctuations
}

\author{
Anna Nie \\ The School of Economics, Shanghai University, Shanghai, China \\ Email address: \\ m18800200686@163.com
}

\section{To cite this article:}

Anna Nie. Using the OLS Method to Analyze Indian Currency Fluctuations. International Journal of Economic Behavior and Organization. Vol. 3, No. 6, 2015, pp. 89-96. doi: 10.11648/j.ijebo.20150306.13

\begin{abstract}
This article mainly uses the OLS method to analyze Indian currency fluctuations in 2014 . We choose the exchange rate fluctuation of the Indian rupee against the Swiss franc as our research object. In order to analyze the influence of the Indian rupee against the Swiss franc, here this article mainly considers two factors: the exchange rate of dollar against the Swiss franc and exchange rate of the euro against the Swiss franc. This article use binary regression model considering two factors affect rupees/Swiss franc. By contrast, we found that the results of 2006 in the original model are better than the 2014.
\end{abstract}

Keywords: OLS, Exchange Rate, India

\section{Introduction}

The exchange rate always plays an important role in the economic life. It is closely related with the national macroeconomic regulation and control, economic policy, and the rate of inflation, even the nation's internal and external economy and international economy. It also plays an important role in the macroeconomic regulation and control, and economic stability. Meanwhile, it also concerns with the economic development trend, and has a significant and profound impact on the economy. Because the exchange rate system structure of India and China have some similarities, so in this paper, we study the Indian currency fluctuations and it should has the some enlightenment on China's exchange rate system. India is one of the fastest growing countries in the world, and has remarkable economic growth. If measured in the same purchasing power, India's gross domestic product is $\$ 4.457$ million in 2011 , with Japan tied for third in the world, second only to the United States, China But if we use the dollar assessment, India's gross domestic product is only $\$ 1.676$ million, ranked 11th in the world, and is nearly the same as the developed countries with just 23 million people in Australia. India's annual growth rate is $5.8 \%$, and $6.1 \%$ in 2011 to 2012.Social wealth in a developing country like India is extreme imbalance, because $10 \%$ of the country's population control $33 \%$ of the nation's income. Because of India's population, the average gross national product (GNP) is very low, in 2011, India's average GDP (PPP) of \$3694, 129th in the world. The dollar exchange rate is $\$ 1389$, for the 140th in the world. Before 1991, influenced by the socialist planned economy, economic policy adopted trade protectionism, the Indian government interference labor and financial markets and regulatory business activities, because of India's economic crisis of 1991, with the collapse of the Soviet union, the new congress government made India begin to implement economic liberalization, by foreign trade and direct investment, gradually transition to the free market, India's economy obtained faster growth.

The Indian economy mainly depends on farming, modern agriculture, handicraft industry, modern industry and its supporting industries primarily. The country still has a population of a quarter could not get enough food and clothing. India has enough foreign exchange reserves, exchange rate stability, and the future official will exchange controls, which is decided by market value. India's manufacturing exports have begun to decline; power supply across large parts of the country is still insufficient. The population of India has a lot of people who is proficient in English, and now is the main, in the $21 \mathrm{st}$ century, information service industry of the world's main producers, computer software exporter and numerous software engineer of the motherland. 


\section{Econometric Analysis Method and Data}

\subsection{Variable Selection}

The choice of samples in this article is based on research needs and data quality.

\subsection{The Selection of Model Indicators}

In order to analyze the influence of the Indian rupee against the Swiss franc, here this article mainly considers two factors: the exchange rate of dollar against the Swiss franc and exchange rate of the euro against the Swiss franc. This article use binary regression model considering two factors affect rupees/Swiss franc.

\subsubsection{Explained Variable Selection}

From the perspective of international literature, the measure of exchange rate fluctuation need choose one of the countries' currencies as a comparison. In this paper, we select Swiss Franc as a comparison of exchange rate fluctuation, because The Swiss Franc is a relatively stable currency.

\subsubsection{Explanatory Variables Selection}

The exchange rate fluctuation of Indian rupee against the Swiss franc is influenced by many factors. Here we consider two main factors: the dollar exchange rate against the Swiss franc, and the euro exchange rate against the Swiss franc. The dollar in the global financial system and the trading system is the primary reserve currency, so he's exchange rate fluctuation has certain impact on the exchange rate fluctuations of other countries around the world, including India. The euro, as another second only to a strong dollar, the currency exchange rate volatility also has a certain impact on Indian rupee against the Swiss franc Through these indicators, the exchange rate fluctuation of Indian rupee against the Swiss franc can be observed through the exchange rate of dollar against the Swiss franc and exchange rate of the euro against the Swiss franc.

\subsubsection{The Selection of Control Variable}

The main content of this article is to study the influence factors of the Indian currency fluctuations over the past few years. In order to eliminate the influence of other factors on the explanatory variables, this article selects the two control variables related to the exchange rate fluctuation to ensure the accuracy of the results.

The fluctuations of Indian exchange rate is influenced by the exchange rate of dollar against the Swiss franc and the exchange rate of the euro against the Swiss franc. Generally, the fluctuations of Indian rupee against Swiss francs are more vulnerable to the influence of the international strong currency.

The exchange rate fluctuations of Indian rupee against the Swiss franc and the exchange rate of dollar against the Swiss franc and exchange rate of the euro against the Swiss franc have close relationship.

\section{The Construction of Regression Model}

\subsection{The Construction of Regression Model of Indian}

$$
\mathrm{Y}=\beta_{0}+\beta_{1} \mathrm{X}_{1}+\beta_{2} \mathrm{X}_{2}+\mathrm{U}_{\mathrm{i}}
$$

\subsection{Data Sampling}

Table 1. The exchange rate of rupee against the Swiss franc, the euro, the dollar.

\begin{tabular}{llll}
\hline Year & INR/SF(Y) & USD/SF( $\left.\mathbf{X}_{1}\right)$ & EUR/SF( $\left.\mathbf{X}_{2}\right)$ \\
\hline $2013-12$ & 0.01444 & 0.8942 & 1.2249 \\
$2014-1$ & 0.01455 & 0.9035 & 1.2312 \\
$2014-2$ & 0.01436 & 0.8942 & 1.2213 \\
$2014-3$ & 0.01445 & 0.8811 & 1.2178 \\
$2014-4$ & 0.01462 & 0.8826 & 1.2191 \\
$2014-5$ & 0.01495 & 0.888 & 1.2204 \\
$2014-6$ & 0.015 & 0.896 & 1.2181 \\
$2014-7$ & 0.01493 & 0.8969 & 1.215 \\
$2014-8$ & 0.01495 & 0.9098 & 1.2219 \\
$2014-9$ & 0.01536 & 0.9354 & 1.2077 \\
$2014-10$ & 0.01552 & 0.9526 & 1.2078 \\
$2014-11$ & 0.01565 & 0.9646 & 1.2026 \\
$2014-12$ & 0.01556 & 0.9759 & 1.2024 \\
$2013-12$ & 0.01444 & 0.8942 & 1.2249 \\
$2014-1$ & 0.01455 & 0.9035 & 1.2312 \\
$2014-2$ & 0.01436 & 0.8942 & 1.2213 \\
$2014-3$ & 0.01445 & 0.8811 & 1.2178 \\
\hline
\end{tabular}

Table 2. The difference of exchange rate.

\begin{tabular}{llll}
\hline Year & $\mathbf{Y}$ & $\mathbf{X}_{\mathbf{1}}$ & $\mathbf{X}_{\mathbf{2}}$ \\
\hline $2014-1$ & 0.003301 & -0.00449 & 0.002228 \\
$2014-2$ & -0.00569 & -0.00449 & -0.00351 \\
$2014-3$ & 0.002517 & -0.00641 & -0.00125 \\
$2014-4$ & 0.005314 & 0.000739 & 0.000463 \\
$2014-5$ & 0.009506 & 0.002649 & 0.000463 \\
$2014-6$ & 0.001509 & 0.003895 & -0.00082 \\
$2014-7$ & -0.00201 & 0.000436 & -0.00111 \\
$2014-8$ & 0.000413 & 0.006202 & -0.00111 \\
$2014-9$ & 0.011984 & 0.012051 & -0.00151 \\
$2014-10$ & 0.004385 & 0.007913 & $3.6 \mathrm{E}-05$ \\
$2014-11$ & 0.003538 & 0.005437 & -0.00187 \\
$2014-12$ & -0.00236 & 0.005058 & $-7.2 \mathrm{E}-05$ \\
Average Value & 0.002701 & 0.002415 & -0.00067 \\
\hline
\end{tabular}

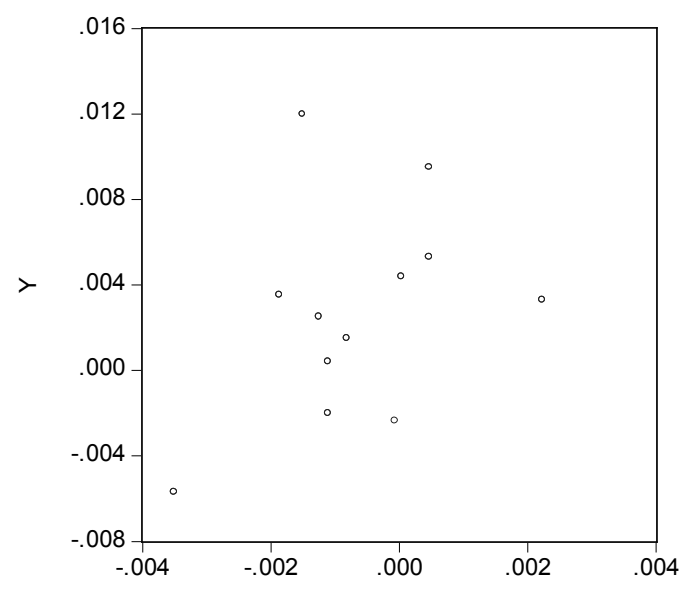

$\mathrm{X} 2$ 


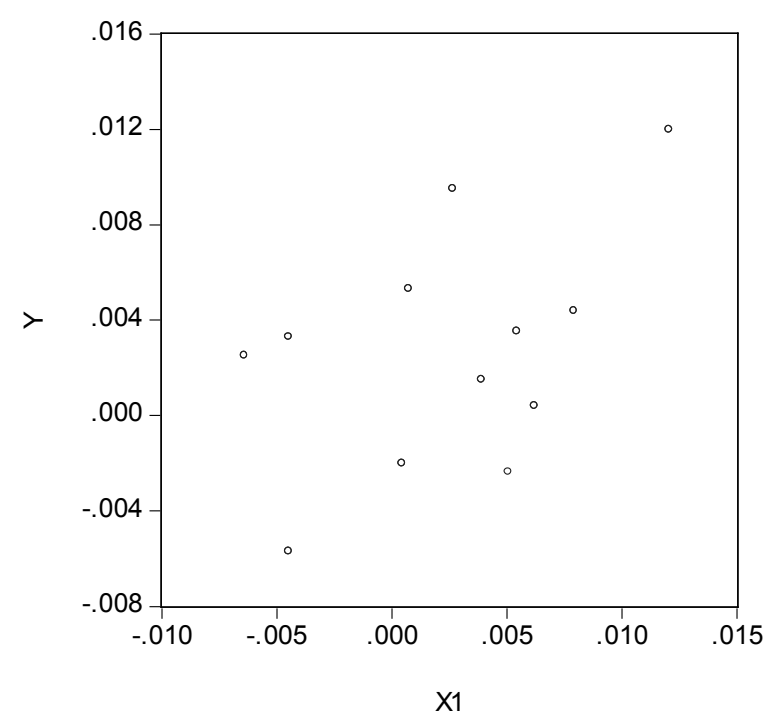

Figure 1. The scatter plot of the correlation between $X_{1}$ and $Y$.

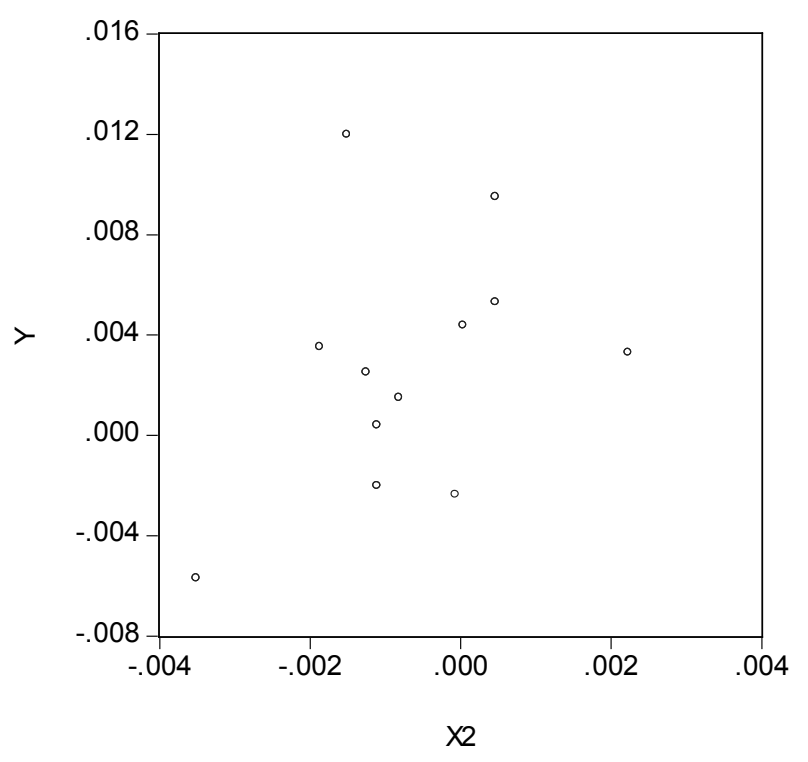

Figure 2. The scatter plot of the correlation between $X_{2}$ and $Y$.

Table 3. Main index regression results.

\begin{tabular}{lll}
\hline$\dot{\boldsymbol{Y}}$ & $\dot{\boldsymbol{X}}_{1}$ & $\dot{\boldsymbol{X}}_{2}$ \\
\hline 0.0006 & -0.00691 & 0.002899 \\
-0.00839 & -0.00691 & -0.00284 \\
-0.00018 & -0.00882 & -0.00058 \\
0.002613 & -0.00168 & 0.001134 \\
0.006805 & 0.000234 & 0.001134 \\
-0.00119 & 0.00148 & -0.00015 \\
-0.00471 & -0.00198 & -0.00044 \\
-0.00229 & 0.003787 & -0.00044 \\
0.009283 & 0.009636 & -0.00084 \\
0.001684 & 0.005498 & 0.000707 \\
0.000837 & 0.003021 & -0.0012 \\
-0.00506 & 0.002643 & 0.000599 \\
\hline
\end{tabular}

Table 4. Main index regression results.

\begin{tabular}{lll}
\hline$\dot{\boldsymbol{Y}}^{2}$ & $\dot{\boldsymbol{X}}_{1}^{2}$ & $\dot{\boldsymbol{X}}_{2}^{2}$ \\
\hline $3.605 \mathrm{E}-07$ & $4.77295 \mathrm{E}-05$ & $8.40337 \mathrm{E}-06$ \\
$7.04019 \mathrm{E}-05$ & $4.77295 \mathrm{E}-05$ & $8.03877 \mathrm{E}-06$ \\
$3.37028 \mathrm{E}-08$ & $7.78767 \mathrm{E}-05$ & $3.31166 \mathrm{E}-07$ \\
$6.82995 \mathrm{E}-06$ & $2.81152 \mathrm{E}-06$ & $1.2863 \mathrm{E}-06$ \\
$4.63137 \mathrm{E}-05$ & $5.45782 \mathrm{E}-08$ & $1.28531 \mathrm{E}-06$ \\
$1.41987 \mathrm{E}-06$ & $2.18963 \mathrm{E}-06$ & $2.20264 \mathrm{E}-08$ \\
$2.21896 \mathrm{E}-05$ & $3.91837 \mathrm{E}-06$ & $1.8982 \mathrm{E}-07$ \\
$5.23304 \mathrm{E}-06$ & $1.43388 \mathrm{E}-05$ & $1.92103 \mathrm{E}-07$ \\
$8.61818 \mathrm{E}-05$ & $9.28547 \mathrm{E}-05$ & $7.00359 \mathrm{E}-07$ \\
$2.83726 \mathrm{E}-06$ & $3.02277 \mathrm{E}-05$ & $4.99919 \mathrm{E}-07$ \\
$7.01267 \mathrm{E}-07$ & $9.12848 \mathrm{E}-06$ & $1.44641 \mathrm{E}-06$ \\
$2.56095 \mathrm{E}-05$ & $6.98487 \mathrm{E}-06$ & $3.58688 \mathrm{E}-07$ \\
\hline
\end{tabular}

Table 5. Main index regression results.

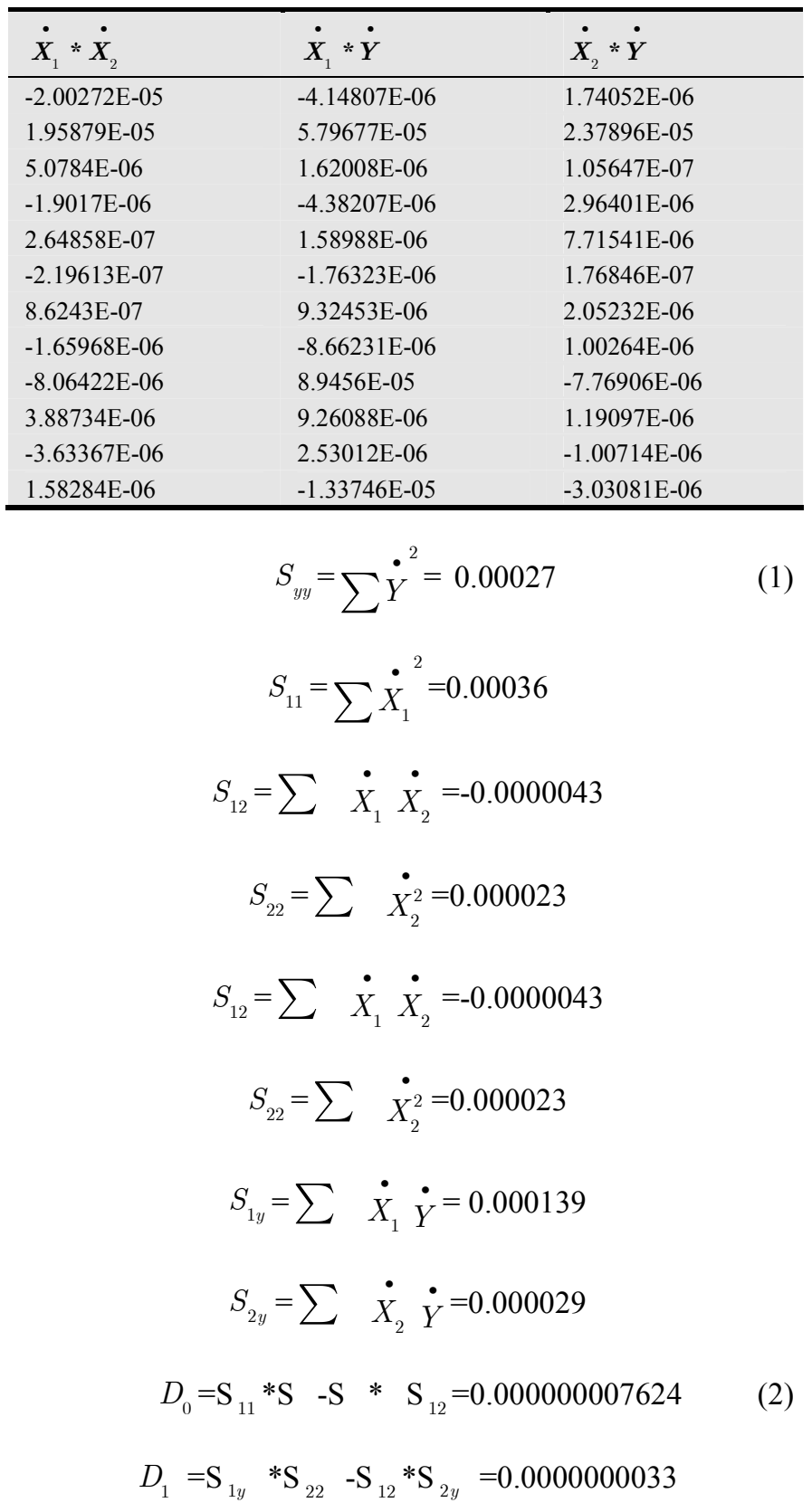




$$
\begin{gathered}
D_{2}=\mathrm{S}_{11} * \mathrm{~S}_{2 y}-\mathrm{S}_{12} * \mathrm{~S}_{1 y}=0.0000000103 \\
\beta_{1}=\frac{D_{1}}{D_{0}}=0.4321 \\
\beta_{2}=\frac{D_{2}}{D_{0}}=1.3516 \\
\beta_{0}=0.002564
\end{gathered}
$$

\subsection{Regression Results and Analysis}

$$
\mathrm{Y}=0.002564+0.4321 X_{1}+1.3516 X_{2}
$$

\section{Model Test}

\subsection{Significance Test}

According the regression equation, we can see the Rupee/Swiss franc is influenced by dollar/Swiss franc, and the euro/Swiss franc. On the surface, the three have positive correlation.

\subsection{Statistical Tests}

\subsubsection{Test of Goodness of Fit}

$$
\begin{gathered}
\mathrm{R}^{2}=\frac{\beta_{1} S_{1 y}+\beta_{2} s_{2 y}}{S_{y y}}=0.3706 \\
R^{2}=1-\frac{n-1}{n-k-1}\left(1-\mathrm{R}^{2}\right)=0.2308
\end{gathered}
$$

Conclusion: the goodness-of-fit is 0.3706 , and the adjusted coefficient is 0.2308 , which shows that the fitting degree of this model on the whole is very poor, explain variables cannot be explained well, simply because there may be a heteroscedasticity, multicollinearity, or the autocorrelation.

\subsubsection{F Test}

Table 6. F test

\begin{tabular}{llll}
\hline $\boldsymbol{Y}_{\boldsymbol{i}}$ & $\boldsymbol{Y}_{\boldsymbol{i}}-\overline{\boldsymbol{Y}}$ & $\left(\boldsymbol{Y}_{i}-\overline{\boldsymbol{Y}}\right)^{2}$ & $\boldsymbol{Y}_{i}-\overline{\boldsymbol{Y}}$ \\
\hline 0.003634 & 0.000933 & $8.69683 \mathrm{E}-07$ & 0.0006 \\
-0.00412 & -0.00682 & $4.64812 \mathrm{E}-05$ & -0.00839 \\
-0.00189 & -0.00459 & $2.108 \mathrm{E}-05$ & -0.00018 \\
0.00351 & 0.000809 & $6.53868 \mathrm{E}-07$ & 0.002613 \\
0.004335 & 0.001633 & $2.66828 \mathrm{E}-06$ & 0.006805 \\
0.00314 & 0.000439 & $1.92759 \mathrm{E}-07$ & -0.00119 \\
0.001257 & -0.00144 & $2.08576 \mathrm{E}-06$ & -0.00471 \\
0.003745 & 0.001044 & $1.08923 \mathrm{E}-06$ & -0.00229 \\
0.005734 & 0.003033 & $9.2007 \mathrm{E}-06$ & 0.009283 \\
0.006032 & 0.003331 & $1.1098 \mathrm{E}-05$ & 0.001684 \\
0.002381 & -0.00032 & $1.02456 \mathrm{E}-07$ & 0.000837 \\
0.004652 & 0.001951 & $3.80757 \mathrm{E}-06$ & -0.00506 \\
\hline
\end{tabular}

Table 7. F test.

\begin{tabular}{lll}
\hline$\left(\boldsymbol{Y}_{i}-\overline{\boldsymbol{Y}}\right)^{2}$ & $\boldsymbol{U}_{i}=\boldsymbol{Y}_{i}-\hat{\boldsymbol{Y}}$ & $\boldsymbol{U}^{2}{ }_{i}=\left(\boldsymbol{Y}_{i}-\hat{\boldsymbol{Y}}\right)^{2}$ \\
\hline $3.605 \mathrm{E}-07$ & -0.00033 & $1.10932 \mathrm{E}-07$ \\
$7.04 \mathrm{E}-05$ & -0.00157 & $2.46908 \mathrm{E}-06$ \\
$3.37 \mathrm{E}-08$ & 0.004409 & $1.94349 \mathrm{E}-05$ \\
$6.83 \mathrm{E}-06$ & 0.001805 & $3.25661 \mathrm{E}-06$ \\
4.631E-05 & 0.005172 & $2.67446 \mathrm{E}-05$ \\
1.42E-06 & -0.00163 & $2.65882 \mathrm{E}-06$ \\
2.219E-05 & -0.00327 & $1.06673 \mathrm{E}-05$ \\
5.233E-06 & -0.00333 & $1.11004 \mathrm{E}-05$ \\
8.618E-05 & 0.00625 & $3.90623 \mathrm{E}-05$ \\
$2.837 \mathrm{E}-06$ & -0.00165 & $2.71562 \mathrm{E}-06$ \\
7.013E-07 & 0.001158 & $1.33983 \mathrm{E}-06$ \\
2.561E-05 & -0.00701 & $4.91781 \mathrm{E}-05$ \\
\hline
\end{tabular}

$$
\begin{aligned}
\mathrm{ESS} & =\sum\left(Y_{i}-Y^{2}=0.00009933\right. \\
\mathrm{RSS} & =\sum\left(Y_{i}-Y_{i}\right)^{2}=0.000168738 \\
\mathrm{~F} & =\frac{E S S / K}{R S S /(N-K-1)}=2.65
\end{aligned}
$$

Hypothesis:

$\mathrm{H}_{0}: \beta_{i}=0$

$\mathrm{H}_{1}$ : not all the $\beta_{i}$ is equal to 0

Statistical magnitude $\mathrm{F}=2.65$ obey the $\mathrm{F}$-distribute whose degrees of freedom is $(2,9)$, according to the table, the critical value is $\mathrm{F}_{\alpha=0.01}=3.01, \mathrm{~F}<3.01$, we cannot refuse the null hypothesis. This model is false under the significance level of 99\%.

\subsubsection{T Test}

$$
\mathrm{S}^{2}=\frac{\sum_{i=1}^{n} u_{i}^{2}}{n-k-1}=0.00001874
$$

$\mathrm{S}_{\partial}^{2}=\frac{S^{2}}{n}+\frac{\bar{X}_{1} S_{22}+\bar{X}_{2} S_{11}-2 \bar{X}_{1} \bar{X}_{2} S_{22}}{D_{0}}=0.000002229$

$$
\begin{gathered}
\mathrm{S}_{\beta_{1}}^{2}=\frac{S^{2} S_{11}}{D_{0}}=0.5598 \\
\mathrm{~S}_{\beta_{2}}{ }^{2}=\frac{S^{2} S_{22}}{D_{0}}=0.8250 \\
\mathrm{~S}_{\alpha}=0.001493 \\
\mathrm{~S}_{\beta_{1}}=0.2366
\end{gathered}
$$




$$
\begin{aligned}
& \mathrm{S}_{\beta_{2}}=0.9083 \\
& \mathrm{t}_{\alpha}=\frac{\alpha}{S_{\alpha}}=1.718041 \\
& \mathrm{t}_{\beta_{1}}=\frac{\beta_{1}}{S}=1.82 \\
& \mathrm{t}_{\beta_{2}}=\frac{\beta_{1}}{S \beta_{2}}=1.4873
\end{aligned}
$$

Hypothesis:

$$
\begin{aligned}
& \mathrm{H}_{0}: \beta_{1}=\beta_{2}=0 \\
& \mathrm{H}_{1}: \text { not all the } \beta_{i} \text { is equal to } 0 \\
& \alpha=0.01
\end{aligned}
$$

According to the table, $\mathrm{t}_{0.01}(9)=2.821, \mathrm{t}_{\alpha}<2.821, \mathrm{t}_{\beta_{1}}$

$<2.821, \mathrm{t}_{\beta_{2}}<2.821$ we cannot reject the null hypothesis. This model is false under the significance level of $99 \%$. The reason may be a heterosexuality, multidisciplinary, or the auto-correlation.

\subsection{Economic Test}

\subsubsection{Auto-correlation Test}

In this article, we choose D-W test to test auto-correlation, which is a regular way to test it.

(1) $\mathrm{H}_{0}: \mathrm{d}=2, \mathrm{H}_{1}: \mathrm{d} \neq 2$

(2) $\mathrm{d}=\frac{\sum_{t=2}^{n}\left(u_{t}-u_{t-1}\right)^{2}}{\sum_{t=1}^{n} u_{t}^{2}}=2.2658$, according to $\mathrm{k}=2, \mathrm{n}=12 \mathrm{we}$

know $\mathrm{d}_{l}=0.569, \mathrm{~d}_{u}=1.274$, meanwhile $\mathrm{d}_{u}<\mathrm{d}<4-\mathrm{d}_{u}$, so we accept the null hypothesis and firmly believe the disturbance is out of auto-correlation.

\subsubsection{Test for Multi-collinearity}

There are many ways to test the multi-collinearity, such as the correlation coefficient test and the variance inflation factor test. In this article, we adopt the first one to test the multi-collinearity. By calculating, we come into conclusion that there is no multi-collinearity. And the counting process is as follows:

$$
\rho=\frac{\operatorname{cov}\left(X_{1}, X_{2}\right)}{\sqrt{D\left(X_{1}\right) D\left(X_{2}\right)}}=-0.04793<1
$$

\subsubsection{Test for Heterosexuality}

In this test, we adopt Glejser test.

$$
\text { Glejser Test: }\left|U_{i}\right|=\alpha_{0}+\alpha_{1} \mathrm{f}\left(\mathrm{x}_{i}\right)+\varepsilon_{i}
$$

(1) Null hypothesis: $\mathrm{H}_{0}: \alpha_{1}=0$,Alternate hypothesis: $\mathrm{H}_{1}$ : $\alpha_{1} \neq 0$

(2) Select $X_{1}$ and use the OLS method to calculate their goodness of fit when $\mathrm{f}(\mathrm{x})=\mathrm{x}_{1}, \mathrm{x}_{1}{ }^{2}, \mathrm{x}_{1}{ }^{3}$, so the results are $\mathrm{R}_{1}$ $=0.12355, \mathrm{R}_{2}=0.1518, \mathrm{R}_{3}=0.6656$

(3) Select $X_{2}$ and use the OLS method to calculate their goodness of fit when $\mathrm{f}(\mathrm{x})=\mathrm{x}_{2}, \mathrm{x}_{2}{ }^{2}, \mathrm{x}_{2}{ }^{3}$, so the results are $\mathrm{R}_{1}=0.2343, \mathrm{R}_{2}=0.3555, \mathrm{R}_{3}=0.01413$

(4) Choose the biggest number of the goodness of fit. We know the biggest number of $\mathrm{R}$ is $\mathrm{f}(\mathrm{x})=\mathrm{x}_{1}{ }^{3}$, at the same time, $\alpha_{1}$ is not equal te 0 , so there don't exist heterosexuality.

(5) Solution: In this article we use the weighted least square method and the weight is $\mathrm{W}_{i}=\frac{1}{\left|u_{i}\right|}$

Table 8. Weighted data.

\begin{tabular}{lll}
\hline $\mathbf{W}_{i} \mathbf{Y}$ & $\mathbf{W}_{i} \mathbf{X}_{\mathbf{1}}$ & $\mathbf{W}_{i} \mathbf{X}_{\mathbf{2}}$ \\
\hline 10.00167879 & -13.61663939 & 6.751430303 \\
-3.621180892 & -2.862096178 & -2.233280255 \\
0.570860059 & -1.45372284 & -0.28269154 \\
2.943916898 & 0.409264266 & 0.256710249 \\
1.837907773 & 0.512189288 & 0.089494973 \\
0.925608589 & 2.389597546 & -0.502611043 \\
-0.614763303 & 0.133337615 & -0.338429664 \\
0.124099099 & 1.862434835 & -0.333180781 \\
1.91738592 & 1.92822928 & -0.24123504 \\
2.657718788 & 4.795886061 & 0.021793333 \\
3.054865285 & 4.694887737 & -1.618157168 \\
-0.336309272 & 0.721549215 & -0.010271041 \\
\hline
\end{tabular}

Table 9. Weighted data processing.

\begin{tabular}{llll}
\hline$\dot{\boldsymbol{Y}}$ & $\dot{\boldsymbol{X}}_{1}$ & $\dot{\boldsymbol{X}}_{2}$ & $\dot{\boldsymbol{Y}}^{2}$ \\
\hline 8.379863 & -13.5762 & 6.621466 & 70.22211 \\
-5.243 & -2.82167 & -2.36324 & 27.48901 \\
-1.05096 & -1.4133 & -0.41266 & 1.104508 \\
1.322101 & 0.449688 & 0.126746 & 1.747952 \\
0.216092 & 0.552613 & -0.04047 & 0.046696 \\
-0.69621 & 2.430021 & -0.63258 & 0.484704 \\
-2.23658 & 0.173761 & -0.46839 & 5.002285 \\
-1.49772 & 1.902858 & -0.46315 & 2.243155 \\
0.29557 & 1.968653 & -0.3712 & 0.087362 \\
1.035903 & 4.83631 & -0.10817 & 1.073095 \\
1.43305 & 4.735311 & -1.74812 & 2.053631 \\
-1.95812 & 0.761973 & -0.14024 & 3.834253 \\
\hline
\end{tabular}


Table 10. Weighted data processing.

\begin{tabular}{lllll}
\hline$\dot{\boldsymbol{X}}_{1}^{2}$ & $\dot{\boldsymbol{X}}_{1}^{2}$ & $\dot{\boldsymbol{Y}} \dot{\boldsymbol{X}}_{1}$ & $\dot{\boldsymbol{Y}} \dot{\boldsymbol{X}}_{2}$ & $\dot{\boldsymbol{X}}_{1} \dot{\boldsymbol{X}}_{2}$ \\
\hline 184.3136 & 43.84381 & -113.767 & 55.48698 & -89.8945 \\
7.961836 & 5.584925 & 14.79402 & 12.39048 & 6.668303 \\
1.997415 & 0.170285 & 1.485315 & 0.433683 & 0.583206 \\
0.202219 & 0.016065 & 0.594533 & 0.167571 & 0.056996 \\
0.305381 & 0.001638 & 0.119415 & -0.00875 & -0.02236 \\
5.905003 & 0.400152 & -1.6918 & 0.440403 & -1.53717 \\
0.030193 & 0.219393 & -0.38863 & 1.0476 & -0.08139 \\
3.62087 & 0.214503 & -2.84994 & 0.69366 & -0.8813 \\
3.875594 & 0.137789 & 0.581875 & -0.10972 & -0.73076 \\
23.38989 & 0.011701 & 5.009948 & -0.11205 & -0.52315 \\
22.42317 & 3.055929 & 6.785936 & -2.50514 & -8.2779 \\
0.580602 & 0.019666 & -1.49204 & 0.274598 & -0.10686 \\
\hline
\end{tabular}

$\beta_{0}=1.3927, \beta_{1}=0.3384, \beta_{2}=1.8679$

So the adjusted model is: $\mathrm{Y}=1.3927+0.3384 \mathrm{X}_{1}+1.8679 \mathrm{X}_{2}$.

\section{Adjusted Model Test}

\subsection{Statistical Test}

\subsubsection{Test of Goodness of Fit}

$$
\begin{aligned}
& \mathrm{R}^{2}=\frac{\beta_{1} S_{1 y}+\beta_{2} s_{2 y}}{S_{y y}}=0.8376 \\
& \bar{R}^{2}=1-\frac{n-1}{n-k-1}\left(1-\mathrm{R}^{2}\right)=0.802
\end{aligned}
$$

The goodness-of-fit rise sharply after adjustment, so the explain variables can well explained variable.

\subsubsection{F Test}

Table 11. F test of adjusted data.

\begin{tabular}{llll}
\hline $\boldsymbol{Y}_{\boldsymbol{i}}$ & $\hat{\boldsymbol{Y}_{\boldsymbol{i}}}-\overline{\boldsymbol{Y}}$ & $\left(\hat{\boldsymbol{Y}_{i}}-\overline{\boldsymbol{Y}}\right)^{2}$ & $\boldsymbol{Y}_{i}-\overline{\boldsymbol{Y}}$ \\
\hline 9.395886786 & 7.774071141 & 60.43618211 & 8.379863144 \\
-5.140232889 & -6.762048533 & 45.72530037 & -5.242996536 \\
-1.020020485 & -2.641836129 & 6.979298133 & -1.050955585 \\
0.61802354 & -1.003792104 & 1.007598589 & 1.322101253 \\
0.340506565 & -1.281309079 & 1.641752957 & 0.216092128 \\
-0.130162606 & -1.75197825 & 3.06942779 & -0.696207055 \\
-0.5870437 & -2.208859345 & 4.879059604 & -2.236578947 \\
0.007921455 & -1.61389419 & 2.604654455 & -1.497716545 \\
0.201937116 & -1.419878528 & 2.016055035 & 0.295570276 \\
1.663730977 & 0.041915333 & 0.001756895 & 1.035903144 \\
-1.433785096 & -3.05560074 & 9.336695881 & 1.433049641 \\
0.225000724 & -1.396814921 & 1.951091922 & -1.958124917 \\
\hline
\end{tabular}

Table 12. F test of adjusted data.

\begin{tabular}{lll}
\hline$\left(\boldsymbol{Y}_{i}-\overline{\boldsymbol{Y}}\right)^{2}$ & $\boldsymbol{U}_{i}=\boldsymbol{Y}_{i}-\boldsymbol{Y}$ & $\boldsymbol{U}^{2}{ }_{i}=\left(\boldsymbol{Y}_{i}-\hat{\boldsymbol{Y}}\right)^{2}$ \\
\hline 70.22210631 & 0.605792002 & 0.36698395 \\
27.48901268 & 1.519051997 & 2.30751897 \\
1.104507642 & 1.590880544 & 2.530900905 \\
1.747951724 & 2.325893358 & 5.409779911 \\
0.046695808 & 1.497401208 & 2.242210377 \\
0.484704264 & 1.055771195 & 1.114652816 \\
5.002285386 & -0.027719602 & 0.000768376 \\
2.24315485 & 0.116177644 & 0.013497245 \\
0.087361788 & 1.715448804 & 2.9427646 \\
1.073095323 & 0.993987811 & 0.988011769 \\
2.053631273 & 4.48865038 & 20.14798224 \\
3.834253189 & -0.561309996 & 0.315068912 \\
\hline
\end{tabular}

$$
\begin{gathered}
\mathrm{ESS}=\sum\left(Y_{i}-Y\right)^{2}=139.6489 \\
\mathrm{TSS}=\sum\left(Y_{i}-Y_{i}\right)^{2}=38.389 \\
\mathrm{~F}=\frac{E S S / K}{R S S /(N-K-1)}=16.3736
\end{gathered}
$$

Suppose: $\mathrm{H}_{0}: \beta_{i}=0 \circ \mathrm{H}_{1}$ :not all the $\beta_{i}$ is equal to $0, \mathrm{~F}>\mathrm{F}$ ${ }_{\alpha=0.01}=3.01$, so we reject the null hypothesis, and failure to reject Alternate hypothesis, that is not all the $\beta_{i}$ is equal to 0 .We can accept the adjusted model on $99 \%$ level.

\subsubsection{T Test}

$$
\begin{aligned}
& \mathrm{S}^{2}=\frac{\sum_{i=1}^{n} u_{i}^{2}}{n-k-1}=4.2645 \\
& \mathrm{~S}_{\partial}^{2}=\frac{S^{2}}{n}+\frac{\bar{X}_{1} S_{22}+\bar{X}_{2} S_{11}-2 \bar{X}_{1} \bar{X}_{2} S_{22}}{D_{0}}=0.3621 \\
& \mathrm{~S}_{\beta_{1}}^{2}=\frac{S^{2} S_{11}}{D_{0}}=0.2315 \mathrm{~S}_{\beta_{2}}^{2}=\frac{S^{2} S_{22}}{D_{0}}=0.0488 \\
& \mathrm{~S}_{\alpha}=0.6017 \mathrm{~S}_{\beta_{1}}=0.4811 \\
& \mathrm{~S}_{\beta_{2}}=0.2193 \mathrm{t}{ }_{\alpha}=\frac{\alpha}{S_{\alpha}^{\wedge}}=3.8462 \\
& \mathrm{t}_{\beta_{1}}=\frac{\beta_{1}}{S}=2.19 \mathrm{t}_{\beta_{2}}=\frac{\beta_{2}}{S \beta_{2}}=8.52
\end{aligned}
$$


Hypothesis: $\mathrm{H}_{0}: \beta_{i}=0, \mathrm{H}_{1}$ :not all the $\beta_{i}$ is equal to $0 . \mathrm{t}_{0.05}$ $(9)=1.833, \mathrm{t}_{\alpha}>1.833, \mathrm{t}_{\beta_{1}}>1.833, \mathrm{t}_{\beta_{2}}>1.833$, so we reject the null hypothesis, and failure to reject alternate hypothesis, that is not all the $\beta_{i}$ is equal to 0 .We can accept the adjusted model on $99 \%$ level.

\subsection{Economic Test: Heteroscedasticity}

There are many ways to test the heteroscedasticity of the model .In this article we choose White test, which is a regular way to test heteroscedasticity. And the detailed process are as follow:

(1) Hypothesis: $\mathrm{H}_{0}: \alpha_{1}=0, \mathrm{H}_{1}: \alpha_{1} \neq 0$

$\mathrm{nR}=3.645$ and $\mathrm{nR}$ obey the $\chi(4)$. Suppose $\alpha=0.05$, then we can get $\chi_{0,05}(4)=9.49, \mathrm{nR}<\chi_{0.05}$, so we cannot reject the null hypothesis, There is no heterosexuality in the model.

(2) Auto-correlation test

$\mathrm{d}=1.3411, \mathrm{~d}_{u}<\mathrm{d}<4-\mathrm{d}_{u}$, So it do not exist auto-correlation.

(3) Multicollinearity test

$\lambda_{1}=292.15328, \quad \lambda_{2}=17.84474, \quad \lambda_{3}=11.29742, \sqrt{C N}=$

$\sqrt{\frac{\lambda_{1}}{\lambda_{3}}}=5.085<10$, So it do not exist multicollinearity.

\subsection{Compare with the 2006's Data}

Table 13. The exchange rate of the countries in 2006

\begin{tabular}{llll}
\hline Month & INR/SF(Y) & USD/SF( $\left.\mathbf{X}_{1}\right)$ & EUR/SF( \\
\hline 1 & -1.53985 & 0.0244446 & 0.107346 \\
2 & -1.53121 & 0.0385407 & 0.115477 \\
3 & -1.53239 & 0.0359498 & 0.115877 \\
4 & -1.54409 & 0.0199882 & 0.108497 \\
5 & -1.57073 & -0.019724 & 0.086182 \\
6 & -1.57219 & -0.011129 & 0.090928 \\
7 & -1.57479 & -0.01104 & 0.092194 \\
8 & -1.57757 & -0.017277 & 0.090293 \\
9 & -1.56896 & -0.009839 & 0.094855 \\
10 & -1.55721 & $-8.69 \mathrm{E}-05$ & 0.100646 \\
11 & -1.55956 & -0.017729 & 0.092229 \\
12 & -1.56719 & -0.038389 & 0.082498 \\
\hline
\end{tabular}

This time we got the results from Eviews: $\mathrm{Y}=-1.7408-0.2242 \mathrm{X}_{1}+1.8626 \mathrm{X}_{2}+\mathrm{U}_{\mathrm{i}}$

\subsubsection{Statistical test}

(1) Test of goodness of fit: $\mathrm{R}^{2}=0.8537$

$$
R^{2=0.8212}
$$

Conclusion: adjusted goodness of fit is larder, which show that the explanatory variable can well explain the explained variable.

(2) F test: Null hypothesis: $\mathrm{H}_{0}: \alpha_{1}=0$,Alternate hypothesis:
$\mathrm{H}_{1}: \alpha_{1} \neq 0 . \mathrm{F}=26.26, \mathrm{~F}(2,9)=10.11, \mathrm{~F}>10.11$. The original assumption is false, so the original equation exists a good linear relationship

(3) $\mathrm{T}$ test: Null hypothesis: $\mathrm{H}_{0}: \alpha_{1}=0$, Alternate hypothesis: $\mathrm{H}_{1}: \alpha_{1} \neq 0, \mathrm{t}_{\alpha}=-15.10, \mathrm{t}_{\beta_{1}}=-0.4156, \mathrm{t} \dot{\beta}_{2}$ $=1.5889$

They are all less than 2.262 when $\alpha=0.025$. So we accept the null hypothesis, because there is no significant linear relationship, the reason may be that there is heteroscedasticity, multicollinearity, or the correlation.

\subsubsection{Economic Test}

(1) D-W test: $d=1.1654$, according to the table, we know that $\mathrm{k}=2, \mathrm{n}=12$ and then get $\mathrm{d}_{l}=0.569, \mathrm{~d}_{u}=1.274, \mathrm{~d}_{l}<\mathrm{d}<\mathrm{d}_{u}$. So the conclusion is there is no autocorrelation.

(2) Test for multi-collinearity

$\lambda_{1}=1.2118 \mathrm{e}+01, \quad \lambda_{2}=3.5869 \mathrm{e}-02, \quad \lambda_{3}=9.4036 \mathrm{e}-04$ $\sqrt{C N}>30$. So the conclusion is there is serious multi-collinearity.

(3) White test: Hypothesis: $\mathrm{H}_{0}: \alpha_{1}=0, \mathrm{H}_{1}: \alpha_{1} \neq 0$ $\mathrm{nR}=10.3179$ and $\mathrm{nR}$ obey $\chi(5)$, if $\alpha=0.05$, then $\chi_{0.1}(5)=9.24, \mathrm{nR}>\chi_{0.1}$, So the conclusion is there is heteroscedasticity on $90 \%$ level .Use the weighted least square method to adjust the original model, and the new model is $\mathrm{Y}=-1.6832+0.1133 \mathrm{X}_{1}+1.2653 \mathrm{X}_{2}$, the adjust goodness of fit is $97 \%$, It pass the $\mathrm{F} 、 \mathrm{~T} 、 \mathrm{D}-\mathrm{W}$ test, but it still exist serious multicollinearity, so we use stepwise regression method to get the final model:

$$
\mathrm{Y}=-1.6935+1.3819 \mathrm{X}_{1}+\mathrm{U}_{\mathrm{i}} \text {. }
$$

\section{Conclusion}

Table 14. Contrast results in 2014 and 2016.

\begin{tabular}{lll}
\hline & $\mathbf{2 0 1 4}$ & $\mathbf{2 0 0 6}$ \\
\hline Goodness of fit & Poor & Good \\
F Test & Don't pass & Pass \\
T Test & Don't pass & Don't pass \\
Heteroskedasticity & Exist & Exist \\
autocorrelation & Don't exist & Don't exist \\
Multicollinearity & Don't exist & Exist \\
\hline
\end{tabular}

By contrast, we can found that the results of 2006 in the original model are better than the 2014 . We analyze the causes in the context of multicollinearity. Before the financial crisis in 2008, the dollar's influence in the world is big, the euro against the dollar dependence is stronger, so the multicollinearity exists. After the 2008 financial crisis, the dollar's influence in the world has shrunk, currencies against the dollar dependence is reduced, and as the Yuan and the yen currency internationalization, further weakening the dollar's influence. Similarly, the dollar and the euro have the similar influence on the influence of the RMB exchange rate. 


\section{References}

[1] Lu Qianjing, Cai Tianyi. India's exchange rate system reform and the enlightenment to China [J]. International Finance Research, 2011, 05: 13-22.

[2] Ma Xianxian, Gong Jian. India's exchange rate regime reform effect and enlightenment [J]. South Asian Studies Quarterly, 2009, 02: 56-60+74+113.

[3] Wu Juan. The compare of China's exchange rate system reform with India and the enlightenment to China [D]. Dongbei University of Finance and Economics, 2012.

[4] Sajal Ghosh. Examining crude oil price - Exchange rate nexus for India during the period of extreme oil price volatility [J]. Applied Energy, 2010, 885.

[5] Natalie D. Hegwood, Hiranya K. Nath. Real exchange rate dynamics: Evidence from India [J]. Economic Analysis and Policy, 2014.

[6] Biswajit Mohanty, N. R. Bhanumurthy. Exchange Rate

Regimes and Inflation: Evidence from India [J]. International Economic Journal, 2014, 282.

[7] Mohsen Bahmani-Oskooee, Rajarshi Mitra. Exchange Rate Risk and Commodity Trade Between the U.S. and India [J]. Open Economies Review, 2008, 191.

[8] Tony Cavoli, Ramkishen S. Rajan. Open economy inflation targeting arrangements and monetary policy rules: Application to India [J]. Indian Growth and Development Review, 2008, 12 .

[9] Hung-Teng Chang. A study on the application of neural network to the interrelationship among Renminbi and foreign currencies [J]. Journal of Information and Optimization Sciences, 2008, 294.

[10] P. Norouzzadeh, B. Rahmani. A multifractal detrended fluctuation description of Iranian rial-US dollar exchange rate [J]. Physica A: Statistical Mechanics and its Applications, 2005, 367.

[11] Zuoquan Zhang, Qin Zhao. The Application of SVMs Method on Exchange Rates Fluctuation [J]. Discrete Dynamics in Nature and Society, 2009, 2009. 